\title{
Radiative Heat and Mass Transfer of an MHD Free Convective Flow of Non-Newtonian Power- Law Fluids Along a Continuously Moving Stretching Sheet with Uniform Surface Heat Flux.
}

\author{
M. A. Samad* and K. C. Saha \\ Department of Mathematics, Dhaka University, Dhaka-1000, Bangladesh.
}

(Received : 9 January 2013; Accepted : 24 September 2013)

\begin{abstract}
An analysis is carried out to study the effects of MHD free convection heat and mass transfer of power-law non-Newtonian fluids along a stretching sheet with thermal radiation. This has been done under the simultaneous action of suction, thermal radiation and uniform transverse magnetic field. The stretching sheet is assumed to continuously moving with a power-law velocity and maintaining a uniform surface heat flux. The governing non-linear partial differential equations governing the flow field for heat and mass transfer problem are transformed into non-linear ordinary differential equations, using similarity transformation, and the resulting problem is solved numerically using Nachtsheim-Swigert shooting iteration technique along with sixth order Runge-Kutta integration scheme. The results from numerical computations have been presented in the from of dimensionless velocity, temperature and concentration profiles, shown graphically and discussed. A parametric study illustrating the influence of the flow field to radiation, buoyancy force, power-law fluid velocity index, Schmidt number, suction or injection parameter and uniform transverse magnetic field on the local skin friction coefficient, the local Nusselt number and the Sherwood number which are of physical and engineering interest are studied and the obtained results are shown graphically and the physical aspects of the problem are discussed. A comparison of the present study is also performed with the previously published work and found excellent agreement.
\end{abstract}

Keywords: MHD, Power-law fluid, Radiation, Stretching sheet, Free convection, Surface heat flux.

\section{Introduction}

Considerable interest has recently been shown in radiation interaction with free convection for heat transfer in fluid. This is due to the significant role of thermal radiation in the surface heat transfer when convection heat transfer is small particularly in free convection problems involving absorbing-emitting fluids. Hot rolling, drawings of plastic films and artificial fibers, glass fiber production, metal extrusion are examples of such physical applications. Many manufacturing processes involve the cooling of continuous sheets or filaments by drawing them through a quiescent fluid which are stretched during the drawing process. The final product of required characteristics depend to a great extent on the rate of cooling which can be controlled by drawing such sheets in an electrically conducting fluid and subject to magnetic field. Sakiadis ${ }^{1}$, first presented boundary layer flow over a continuous solid moving with constant speed. Elbashbeshy ${ }^{2}$ investigated heat transfer over a stretching surface with variable and uniform surface heat flux subject to injection and suction. Vajravelu et al. $^{3}$ studied the convective heat transfer in an electrically conducting fluid near an isothermal stretching sheet and studied the effect of internal heat generation or absorption. Also, Glauert ${ }^{4}$ analyzed magnetohydrodynamic boundary layer on a flat plate.

The study of non-Newtonian fluid flow and heat transfer over a stretched surface gets attention because numerous industrially important fluids exhibit non linear relationship between shear stress and rate of strain such as polymer solution, molten plastics, pulps, paints, and foods. Rajgopal et $a l^{5}{ }^{5}$ studied flow of viscoelastic fluid over stretching sheet. Gupta et $a l^{6}{ }^{6}$ extended the problem to study heat transfer, and Datti et al. ${ }^{7}$ analyzed the problem over a nonisothermal stretching sheet. The MHD boundary layer flow over a continuously moving plate for a micropolar fluid has been studied by Rahman and Sattar ${ }^{8}$ and Raptis ${ }^{9}$. Several authors (e.g. Anderson et. al. ${ }^{10}$, Mahmoud and Mahmoud ${ }^{11}$ and Chen et. al..$^{12}$ ) adopted the non-linearity relation as power-law dependency of shear stress on rate of strain. Recently, C.H.Chen ${ }^{13}$ studied the effect of magnetic field and suction/injection on the flow of power-law nonNewtonian fluid over a power-law stretched sheet subject to a surface heat flux.All the above investigations are restricted to MHD flow and heat transfer problems. However, of late, the radiation effect on MHD flow and heat transfer problems has become more important industrially. Many processes in engineering areas occur at high temperatures and the knowledge of radiative heat transfer becomes very important for the design of the pertinent equipments. Nuclear power plants, gas turbines and the various propulsion devices for aircrafts, missiles, satellites and space vehicles are examples of such engineering areas. There are various kinds of high temperature systems such as a heat exchanger and an internal combustor, in which the radiation may not be negligible in comparison with the conductive and convective heat transfer. During combustion of a hydrocarbon fuel and the particles such as soot and coal suspended in a hot gas flow absorb, emit and scatter the radiation. The interaction of radiation with hydromagnetic flow has become industrially more prominent in the processes wherever high temperatures occur. Hakiem ${ }^{14}$ investigated radiation effect on a heated surface. Takhar et $a l .{ }^{17}$ analyzed the radiation effects on MHD free convection flow past a semi-infinite vertical plate using Runge-Kutta Merson quadrature. Ali et. al. ${ }^{18}$ studied radiation effects on 
free convection flow over a horizontal plate. Chamkha et $a l^{15}$ analyzed radiation effects on free convection flow past a semi-infinite vertical plate.

In the present paper, the problem studied by C. H. Chen ${ }^{13}$ has been extended for the case of free convection to implement the effect of thermal radiation. Here, we have an investigation is carried out to investigate the effects of MHD free convection heat and mass transfer of power-law nonNewtonian fluids along a stretching sheet.

\section{Governing Equations}

Let us consider a steady two dimensional MHD free convection laminar boundary layer flow of a viscous incompressible and electrically conducting fluid obeying the power-law model along a permeable stretching sheet under the influence of thermal radiation. Introducing the Cartesian co-ordinate system, the $\mathrm{X}$-axis is taken along the stretching sheet in the vertically upward direction and the $\mathrm{Y}$-axis is taken normal to the sheet. Two equal and opposite forces are introduced along the X-axis, so that the sheet is stretched. This continuous sheet is assumed to move with a velocity according to a power-law from, i.e. $u_{w}=C x^{p}$ and be subject to a surface heat. The ambient temperature of the flow is $T_{\infty}$ and the concentration of the uniform flow is $C_{\infty}$ The fluid is considered to be gray, absorbing- emitting radiation but non-scattering medium and the Rossland approximation is used to describe the radiactive heat flux in the energy equation. The concentration is assumed to be non-reactive. The radioactive heat flux in the X-direction is considered negligible in the comparison to the Y-direction. A strong magnetic field in comparison to the applied magnetic field. The electrical current flowing in the fluid gives rise to an induced magnetic field if the fluid were an electrical insulator, but here we have taken the fluid to be the electrically conducting. Hence, only the applied magnetic field $B$ plays a role which gives rise to magnetic forces $F_{x}=\frac{\sigma B u}{\rho}$ in the X-direction, where $\sigma$ is the electrical conductivity, $B$ is the magnetic field strength (magnetic induction ) and $\rho$ is the density of the fluid. Under the above assumptions, the governing boundary layer equations are:

$\frac{\partial u}{\partial x}+\frac{\partial v}{\partial y}=0$

$u \frac{\partial u}{\partial x}+v \frac{\partial u}{\partial y}=\frac{K}{\rho} \frac{\partial}{\partial y}\left(\left|\frac{\partial u}{\partial y}\right|^{n-1} \frac{\partial u}{\partial y}\right)+g \beta\left(T-T \partial_{o}-\frac{\sigma B^{2} u}{\rho}\right.$

$u \frac{\partial T}{\partial x}+v \frac{\partial T}{\partial y}=\alpha \frac{\partial^{2} T}{\partial y^{2}}-\frac{1}{\rho c_{p}} \frac{\partial q_{r}}{\partial y}$

$u \frac{\partial C}{\partial x}+v \frac{\partial C}{\partial y}=D_{m} \frac{\partial^{2} C}{\partial y^{2}}$

The radiative heat flux $q_{r}$ is described by the Rosseland approximation such that,

$q_{r}=-\frac{4 \sigma_{1}}{3 k_{1}} \frac{\partial T^{4}}{\partial y}$ where, $\sigma_{1}$ is the Stefan-Boltzman constant and $k_{1}$ is the Rosseland mean absorption coefficient. It is assumed that the temperature difference within the flow is sufficiently small such that $T^{4}$ can be expressed in a Taylor series about the free steam temperature $T_{\text {cand }}$ then neglecting higherorder terms. This results in the following approximation:

$$
T^{4} \approx 4 T_{\infty}^{3} T-3 T_{\infty}^{4}
$$

Using (5) and (6) in the last term of equation (3), we obtain,

$\frac{\partial q_{r}}{\partial y}=-\frac{16 \sigma_{1} T_{\infty}^{3}}{3 k_{1}} \frac{\partial^{2} T}{\partial y^{2}}$

Introducing $q_{r}$ in equation (3), we obtain the following governing boundary layer equations as:

$\frac{\partial u}{\partial x}+\frac{\partial v}{\partial y}=0$

$u \frac{\partial u}{\partial x}+v \frac{\partial u}{\partial y}=\frac{K}{\rho} \frac{\partial}{\partial y}\left(\left|\frac{\partial u}{\partial y}\right|^{n-1} \frac{\partial u}{\partial y}\right)+g \beta\left(T-T \partial_{b}-\frac{\sigma B^{2} u}{\rho}\right.$

$u \frac{\partial T}{\partial x}+v \frac{\partial T}{\partial y}=\alpha \frac{\partial^{2} T}{\partial y^{2}}+\frac{16 \sigma_{1} T_{\infty}^{3}}{3 \rho c_{p} k_{1}} \frac{\partial^{2} T}{\partial y^{2}}$

$u \frac{\partial C}{\partial x}+v \frac{\partial C}{\partial y}=D_{m} \frac{\partial^{2} C}{\partial y^{2}}$

The appropriate boundary conditions are:

$\left.\begin{array}{ccc}u_{w}=C x^{p}, \quad v=v_{w}, \quad \partial T / \partial y=-q_{w} / \kappa, C=C_{\infty}+b x & \text { at } y=0, x>0 \\ u_{w} \rightarrow 0, & T \rightarrow T_{\infty} \quad C \rightarrow C_{\infty} & \text { as } y \rightarrow \infty\end{array}\right\}$

where, $u$ and $v$ are the velocity components, $K$ is the consistency coefficient, $c_{p}$ is the specific heat at constant pressure, $B(x)$ is the magnetic field, $T$ is the temperature of the fluid layer, $g$ is the acceleration due to gravity, $\beta$ is the volumetric coefficient of thermal expansion, $\sigma$ is the electric conductivity, $\rho$ is the density of the fluid, $\alpha$ is the thermal diffusivity, $k$ is the thermal conductivity of the fluid, $n$ is the flow behavior index, and $q_{r}$ is the radiative heat flux. $v_{w}$ is the velocity component at the wall having positive value to indicate suction and negative value for injection, $q_{w}$ is surface heat flux. The power index $p$ indicates surface is accelerated or decelerated for positive and negative values respectively.

\section{Similarity Analysis}

In order to obtain a similarity solution of the problem, we introduce a similarity parameter $(1 x)$ such that $(x)$ is a length scale. We now introduce the following dimensionless variables:

$$
\begin{aligned}
& \eta=\frac{y}{\delta(x)}=\left(\frac{C^{2-n}}{K / \rho}\right)^{1 /(n+1)} x^{\{p(2 n-1)+1\} /(n+1)} y \\
& \psi=\left(\frac{C^{1-2 n}}{K / \rho}\right)^{-1 /(n+1)} x^{\{p(2 n-1)+1\} /(n+1)} f(\eta) \\
& \theta(\eta)=\frac{\left(T-T \not h e_{x}^{1 /(n+1)}\right.}{q_{w} x / \kappa}
\end{aligned}
$$


$\phi(\eta)=\frac{C-C_{\infty}}{b x}$

where,

प) प्र

distance normal to the sheet, $f$ is the dimensionless stream function, $\theta$ and $\phi$ are the dimensionless fluid temperature and concentration respectively. Using the transformations from equations (13) to (16) in equations (9) to (11), we obtain the following dimensionless equations as,

$$
\begin{aligned}
& \left(|f|^{n^{\prime}-1} f\right)^{\prime \prime}+\frac{p(2 n-1)+1}{(n+1)} f f^{\prime \prime}-p(f)^{2}-M f^{\prime}+\lambda \theta=0 \\
& \frac{3 N+4}{3 N P r} \theta^{\prime}+\frac{p(2 n-1)+1}{(n+1)} f \theta^{\prime}+\frac{p(2-n)-1}{(n+1)} f \theta^{\prime}=0 \\
& \frac{1}{S c} \phi^{\prime \prime}+\frac{p(2 n-1)+1}{(n+1)} f \phi^{\prime}-f^{\prime} \phi=0
\end{aligned}
$$

The transformed boundary conditions are,

$f^{\prime}=1, \quad f=\frac{n+1}{p(2 n-1)+1} f_{w}, \quad \theta^{\prime}=-1, \quad \phi=1 \quad$ at $\eta=0 \quad$

$f^{\prime} \rightarrow 0, \quad \theta \rightarrow 0, \quad \phi \rightarrow 0 \quad$ as $\left.\eta \rightarrow \infty\right)$

where, $M=\frac{\sigma B^{2} x}{\rho u_{w}}$ is the magnetic field parameter, $\operatorname{Pr}=$ $\frac{x u_{w}}{\alpha} R e_{x}^{-2 /(n+1)}$ is the generalized Prandtl number, $N=\frac{k k_{1}}{4 \sigma_{1} T_{\infty}^{3}}$ is the radiation number, $f_{w}=-\frac{v_{w}}{u_{w}} R e_{x}{ }^{1 /(n+1)}$ is the suction parameter, $\lambda=\frac{G r}{R e_{x}^{1 /(n+1)}}=\frac{g \beta\left(q_{w} / k\right) x^{2}}{u_{w}{ }^{2}} R e_{x}^{\frac{-1}{(n+1)}}$ is the buoyancy parameter, $S c=\frac{u_{w} x}{D_{m}} R e_{x}^{\frac{-2}{(n+1)}}$ is the local Schmidt number and $R e_{x}=\frac{\rho u_{w}{ }^{2-n} x^{n}}{K}$ is the local Reynolds number.

Here, we note that the magnetic field strength $B$ should be proportional to $x$ to the power of $(p-1) / 2$ to eliminate the dependence of $\mathrm{M}$ on $x$, i.e. $B(x)=B_{0} x^{(p-1) / 2}$, where $B_{0}$ is a constant. The parameters of engineering interest for the present problem are skin friction coefficient $\left(C_{f}\right)$, local Nusselt number $\left(N u_{x}\right)$ and local Sherwood number $\left(S h_{x}\right)$, which indicate physically wall shear stress, local wall heat transfer rate and local wall mass transfer rate respectively. The skin friction coefficient $\left(C_{f}\right)$ is given by,

$$
\begin{aligned}
C_{f} & =\frac{\tau_{w}}{\frac{1}{2} \rho u_{w}{ }^{2}} \\
\text { or, } R e_{x}{ }^{1 /(n+1)} C_{f} & =2\left|f^{\prime \prime}(0)\right|^{n-1} f^{\prime \prime}(0)
\end{aligned}
$$

The local Nusselt number $N u_{x}$ is defined as,

$$
\begin{gathered}
N u_{x}=\frac{h x}{k}=\frac{R e_{x}{ }^{1 /(n+1)}}{\theta(0)} \\
\text { or, } \quad N u_{x} R e_{x}{ }^{-1 /(n+1)}=1 / \theta(0)
\end{gathered}
$$

and the local Sherwood number $(S h)$ is given by

$$
\begin{aligned}
S h & =\frac{x M_{w}}{D_{m}\left(C-C_{\infty}\right)} \\
\text { or, } \quad S h e_{x}^{-1 /(n+1)} & =-\phi^{\prime}(0)
\end{aligned}
$$

Thus from equation (21), (22) and (23), we see that the skin friction coefficient $\left(C_{f}\right)$ local Nusselt number $\left(\mathrm{N} u_{x}\right)$ and Sherwood number $(S h)$ are proportional to $2|f(0)|^{n-1} f^{\prime}(0)$, $1 / \theta(0)$ and $-\phi^{\prime}(0)$ respectively.

\section{Results and Discussion}

The system of transformed governing equations (17)-(19) is solved numerically using Nachtsheim - Swigert [15] shooting iterative technique along with sixth order RungeKutta integration scheme. Now in order to discuss the results, we solve the system (17)-(19) for different physically important parameters and carry out the discussion how these parameters do effect on the velocity, temperature and concentration of the flow field. The effects of suction on the velocity, temperature and concentration profiles are shown in the Fig.1(a), Fig.1(b) and Fig.1(c) respectively. Due to the effect of suction some of the retarded fluid particles are taken out from the boundary layer and thus prevent the boundary layer separation. We observe that the velocity profiles decrease with the increase of suction parameter. This indicates the fact that for a flow field of higher suction, the velocity of the fluid particle is low and velocity stabilizes very quickly (Fig.1(a)) near the stretching sheet. The negative values of $f_{w}$ indicates injection. For, $f_{w}=-1.0$, the velocity increases at first due to buoyant force $(\lambda=2.0)$. The temperature and concentration profiles in Fig.1(b) and Fig.1(c) show a decreasing trend with the increase of suction parameter $\left(f_{w}\right)$. Thus it reduces the thermal and mass boundary layer thickness with the increase of the suction parameter to a significant amount.

In Fig.2 we have plotted the dimensionless velocity, temperature and concentration profiles showing the effect of different values of buoyancy parameter( $\lambda$ ). Fig.2(a) shows the effect of the velocity field. We see that the velocity profiles increase with the increase of the buoyancy parameter $(\lambda)$. The velocity field increases slowly for $\lambda$ at first near the stretching sheet and then decreases monotonically. Fig.2(b) shows the effect of buoyancy parameter $(\lambda)$ on the temperature field. The temperature profiles decrease as the buoyancy parameter $(\lambda)$ increases, and for a fixed value of $\lambda$, the temperature decreases monotonically. It is observed from Fig.2(c) that concentration profiles is strictly decreasing with the increase 
of buoyancy parameter $(\lambda)$. It is clear from Fig.2(a)- Fig.2(c) that the buoyancy force has significant effects on the flow field. As $\lambda$ increases the velocity profiles stabilize more quickly and near $\eta=2.2$, we observed a cross flow in the velocity field (Fig.2(a)). The case $\lambda=0$ corresponds to forced convection flow. We thus see that, the velocity profiles stablize more quickly for forced convection flow.

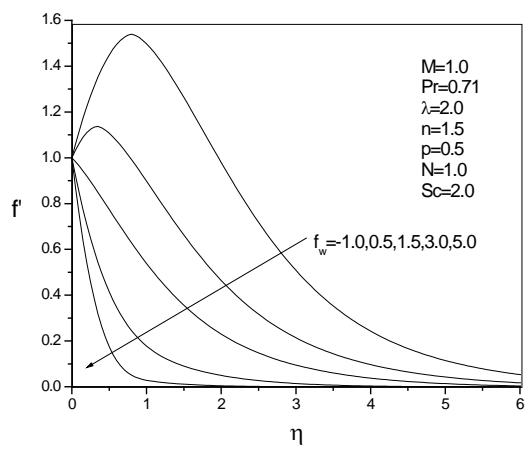

$1(\mathrm{a})$



1(b)

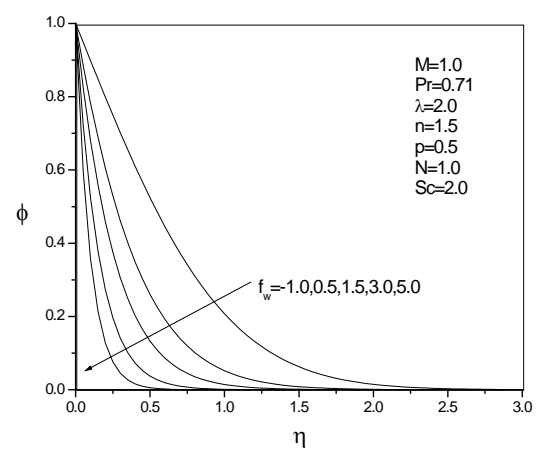

1(c)

Fig. 1. Suction parameter $\left(f_{w}\right)$ effect on (a) velocity, (b) temperature and (c) concentration profile.

In Fig.3, the effect of radiation number $(N)$ on velocity, temperature and concentration profiles are shown respectively. It is quite clear from both the graphs that velocity and temperature profiles decrease as the radiation number $(N)$ increases. However, the velocity profiles rise near the stretching sheet for all values of $N$. It also clears the fact that the velocity profile and wall temperature decreases very rapidly for $N \geq 1.0$ to indicate that the radiation effect can be used to control the velocity and temperature of the boundary layer. The concentration profile in Fig. 3(c), with increase of radiation parameter $(N)$, the concentration in the vicinity of the boundary layer increases. There is a notable difference in the velocity profiles between the values 0.10 and 0.50 for radiation parameter $(N)$. This is because, at the value of 0.10 the radiation is negligible and upon reaching the value of 0.50 , it becomes significant at the velocity field in the velocity boundary layer. With the increase of the radiation parameter $(N)$ the velocity boundary layer thickness reduces rapidly. Due to the radiation from a system, the wall temperature decreases to a great extent. From Fig.3(b), we find that our numerical results agree with the experimental phenomenon. According to the Newton's law of cooling, the rate of heat transfer is thus increased.

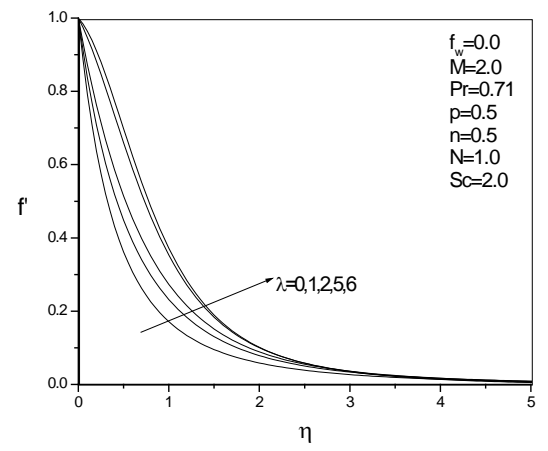

2(a)

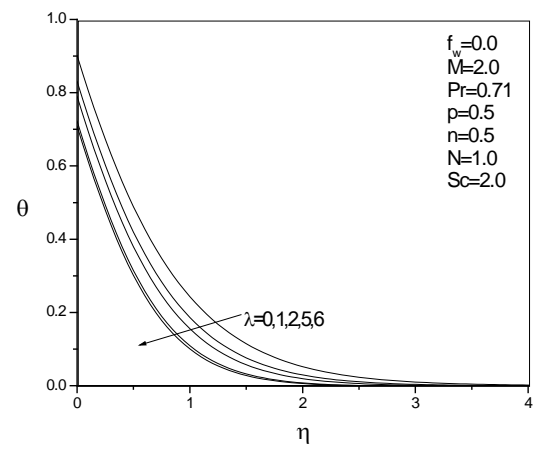

2 (b) 


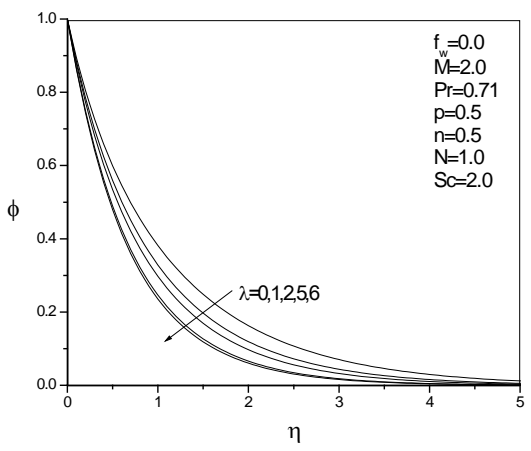

2(c)

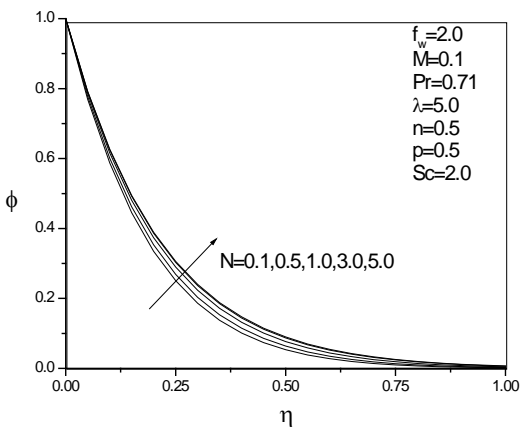

3(c)
Fig. 2. Buoyancy parameter (\) effect on (a) velocity, temperature and (c) concentration profile.

No significant effects are seen on the velocity and temperature in Fig.4(a) and Fig.4(b). In Fig.4(c), the concentration profiles show a sharp variation with the increase of Schmidt number $(S c)$. The decreasing rate of



3(a)

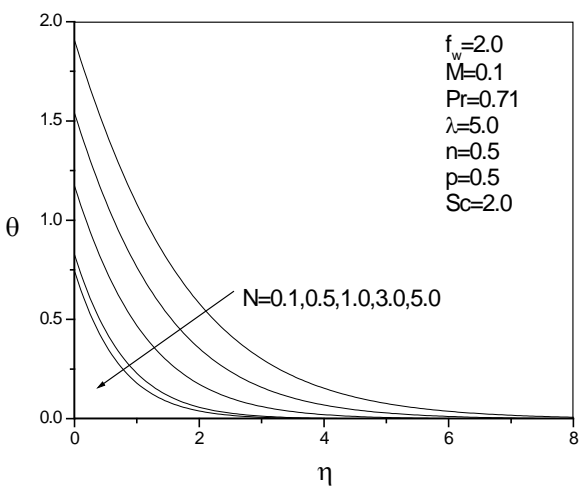

3(b)

(b)
Fig. 3. Radiation number (N) effect on (a) velocity, (b) temperature and (c) concentration profile.

the concentration profiles decrease to a large extent due to the increase in the Schmidt number $(S c)$. Because with the increase of the Schmidt number $(S c)$, the kinematic viscosity increase and the mass diffusion coefficient decrease causing the concentration decrease rapidly.

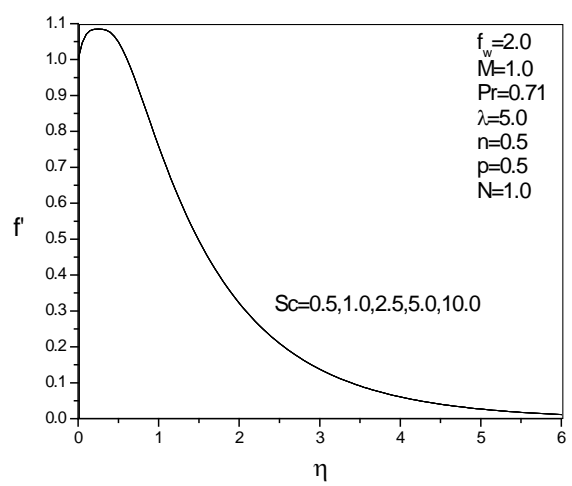

4(a)

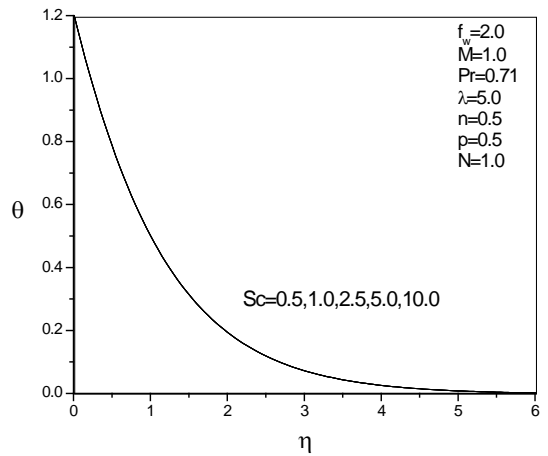

4(b) 


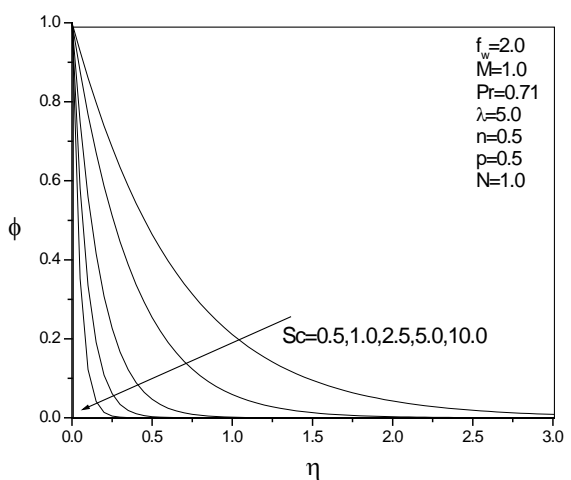

4(c)

Fig. 4. Schmidt number $(S c)$ effect on (a) velocity, (b) temperature and (c) concentration profile.

Fig. 5 expresses the effect of magnetic field parameter $(M)$ on the velocity, temperature and concentration profiles. From Fig.5, we observe that the velocity profiles decrease whereas temperature and concentration profiles increase as the magnetic field parameter $(M)$ increase. From Fig.5(a) the magnetic field produces a retarded action on the velocity field, thus decreasing the velocity at higher rate. The momentum boundary layer thickness decreases slightly with the increase of the magnetic field parameter( $M)$. In Fig.5(b) the heat transfer increase with the increase of the magnetic field strength. This implies that the thermal boundary layer thickness decreases a small amount with the increase of the magnetic field parameter $(M)$. The concentration profiles in Fig.5(c) also show a smaller pattern of decreasing transfer rate due to the magnetic field parameter $(M)$.

In Fig. 6 the effects of Prandtl number $(P r)$ on the velocity, temperature and concentration profiles are shown. Fig.6(a) and Fig.6(b) display the effect of Prandtl number (Pr) on velocity and temperature profiles respectively. From both the figure we observe that the velocity and temperature profiles decrease with the increase of Prandtl number $(\mathrm{Pr})$. However, for $\operatorname{Pr}=0.71$ and 1.5 , there is a rise in the velocity boundary layers near the stretching sheet. Physically $\operatorname{Pr}=0.71$ corresponds to air at $20^{\circ} \mathrm{C}$ and $\operatorname{Pr}=7.0$ corresponds to water at room temperature. From Fig.6(b) we see that for small $\operatorname{Pr}$ wall temperature is very high compared to larger values. From above investigations, it follows that $\operatorname{Pr}$ strongly influences the relative growth of the velocity and thermal boundary layers. On the other hand, in Fig. 6(c) the concentration profiles increase with the increase of Prandlt number $(\mathrm{Pr})$.

The effect of velocity index $(p)$ on velocity, temperature and concentration field are shown in the Fig. 7 for pseudoplastic. It is clear from Fig. 7(a) and Fig. 7(b) that, velocity and temperature profiles increases with increasing velocity index $(p)$ for pseudo-plastic fluids and in the Fig. 7(c) concentration profiles are slightly increase with the increase of the velocity-index $(p)$.

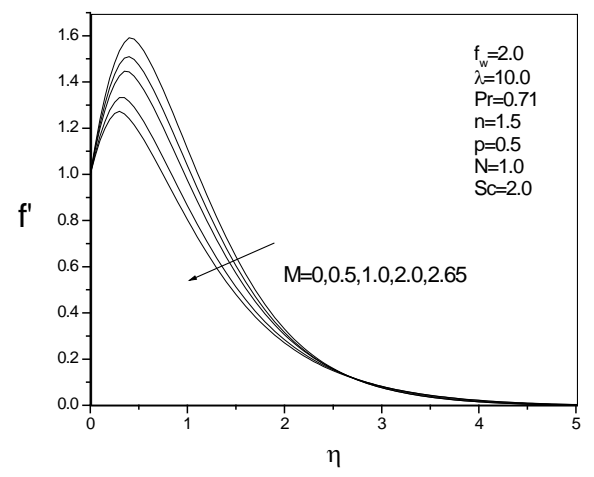

5(a)

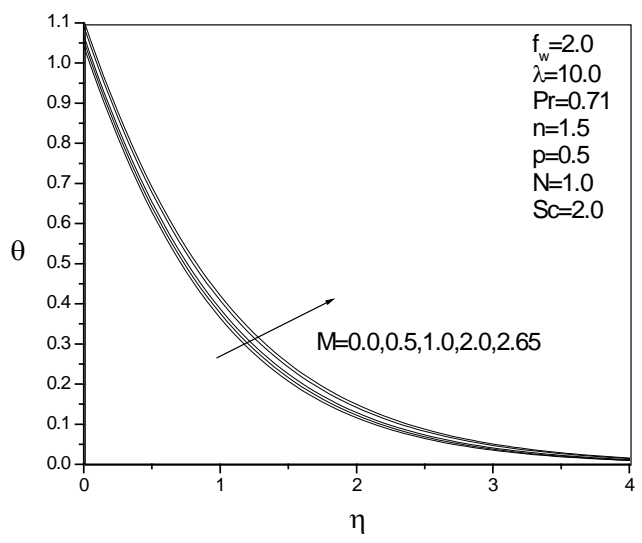

5(b) 


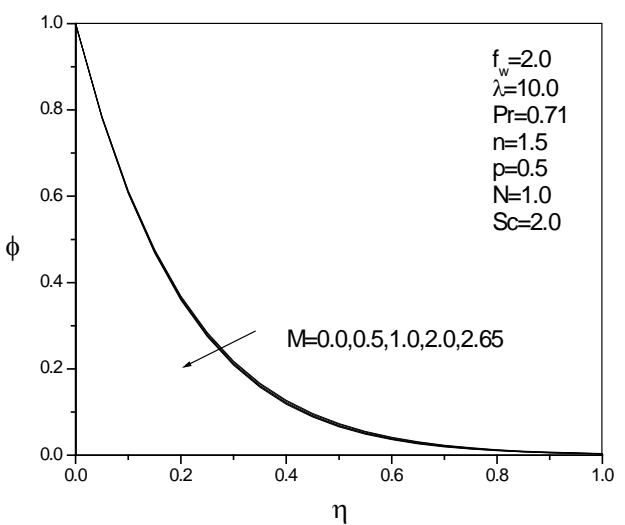

5(c)

Fig. 5. Magnetic field parameter (M) effect on (a) velocity, (b) temperature and (c) concentration profile.

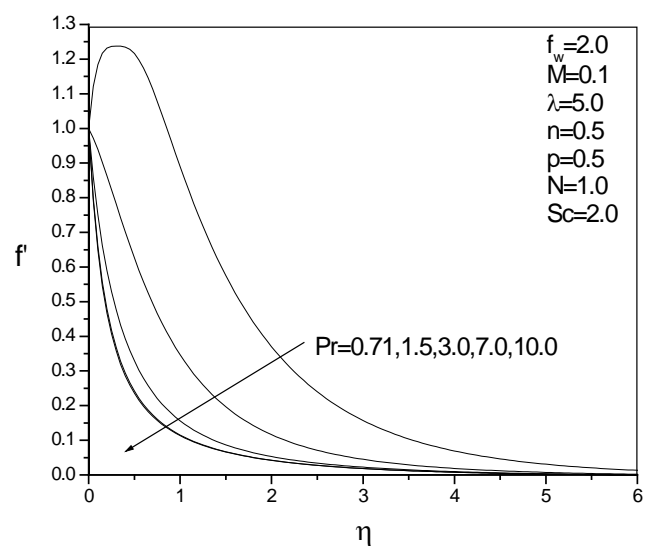

6(a)

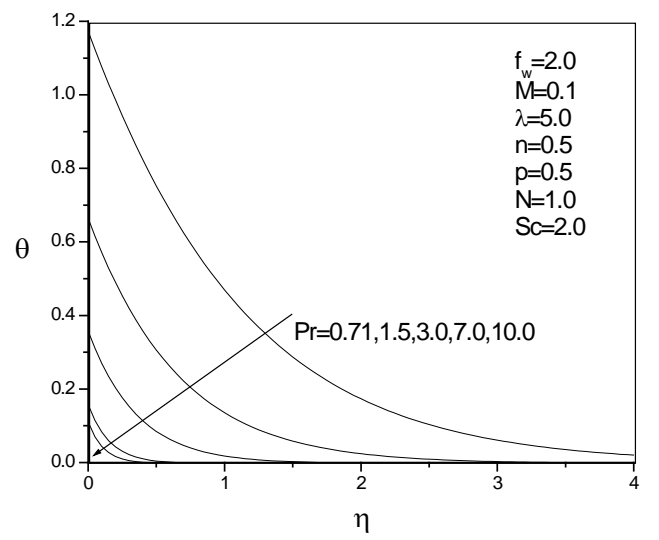

6(b)

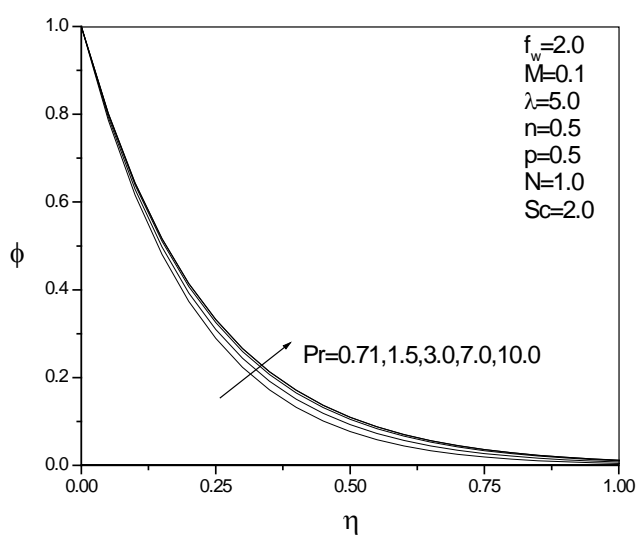

6(c)

Fig. 6. Prandtl number $(\mathrm{Pr})$ effect on (a) velocity, (b) temperature and (c) concentration profile.

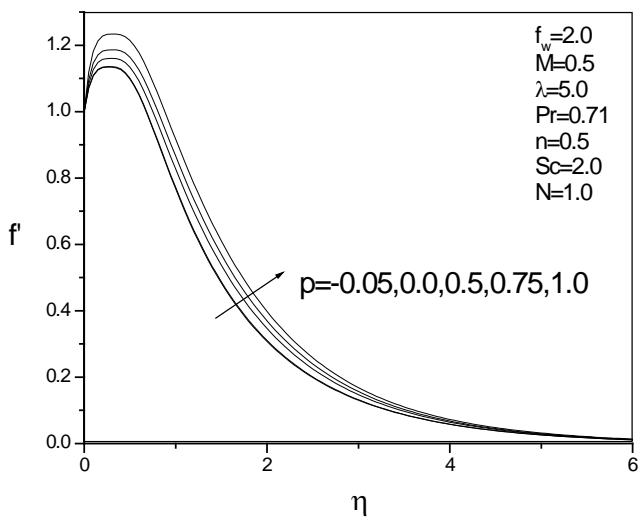

7(a)

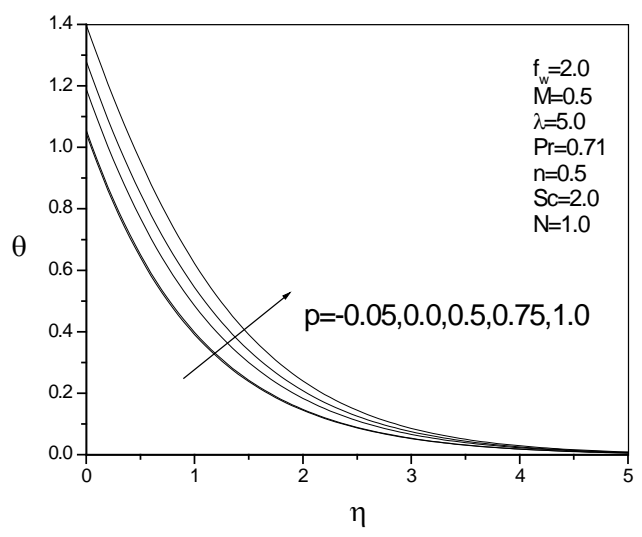

7(b) 


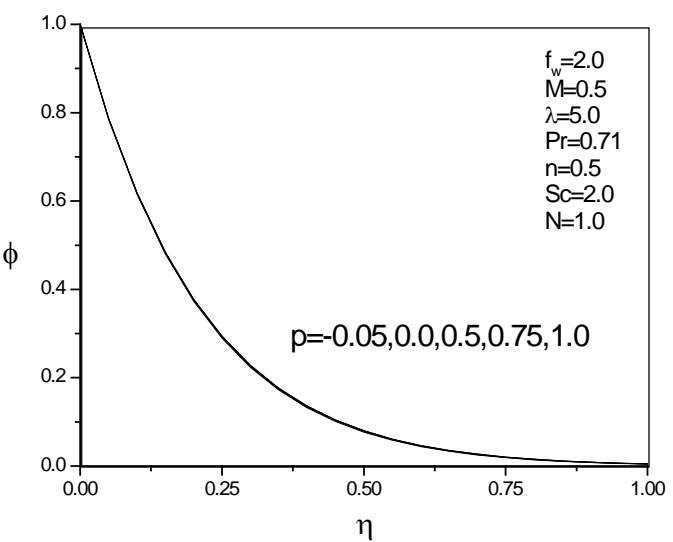

$7(c)$

Fig. 7. Velocity index $(p)$ effect on (a) velocity, (b) temperature and (c) concentration profile for pseudo-plastic fluid.

Table 1 shows the comparison of the skin friction coefficient and the local heat transfer for various values of $n$ and $M$ with $p=0.5$ and $P r=5.0$. As can be seen the results agree well among these two sets. The skin friction coefficient and the local heat transfer rate results have been obtained by Chen ${ }^{13}$ for forced convection (i.e. $\lambda=0$ ) in the absence of the thermal radiation.

Table. 1*. Comparison of $\operatorname{Re}_{x}{ }^{1 /(n+1)} \mathrm{C}_{\mathrm{f}}$ and $N u_{x} \operatorname{Re}_{x}{ }^{-1 /(n+1)}$

\begin{tabular}{|c|c|c|c|c|c|c|}
\hline \multicolumn{3}{|c|}{ Parameters } & \multicolumn{2}{|c|}{$R e_{x}{ }^{1 /(n+1)} C_{f}$} & \multicolumn{2}{|c|}{$N u_{x} R e_{x}^{-1 /(n+1)}$} \\
\hline $\mathbf{n}$ & $\mathbf{M}$ & $f_{w}$ & Chen $^{13}$ & $\begin{array}{c}\text { Present } \\
\text { study }\end{array}$ & Chen $^{13}$ & $\begin{array}{c}\text { Present } \\
\text { study }\end{array}$ \\
\hline \multirow[t]{5}{*}{0.5} & 1 & -0.2 & -2.472213 & -2.4723340 & 0.791413 & 0.7913201 \\
\hline & & 0.0 & -2.633241 & -2.6334386 & 1.346116 & 1.3461035 \\
\hline & & 0.6 & -3.213067 & -3.2137484 & 3.683003 & 3.6833107 \\
\hline & 5 & -0.2 & -3.881351 & -3.8813143 & 0.367234 & 0.3679528 \\
\hline & & 0.6 & -4.608094 & -4.6078741 & 3.528467 & 3.5287845 \\
\hline \multirow[t]{6}{*}{1.0} & 1 & -0.2 & -2.323803 & -2.3238161 & 1.019734 & 1.0198238 \\
\hline & & 0.0 & -2.519363 & -2.5193782 & 1.578424 & 1.5785883 \\
\hline & & 0.6 & -3.198565 & -3.1985827 & 3.866662 & 3.8672224 \\
\hline & 5 & -0.2 & -4.529439 & -4.5294577 & 0.692476 & 0.6935165 \\
\hline & & 0.0 & -4.726210 & -4.7262298 & 1.320497 & 1.3231287 \\
\hline & & 0.6 & -5.366930 & -5.3668243 & 3.754876 & 3.7553935 \\
\hline \multirow[t]{4}{*}{1.5} & 1 & -0.2 & -2.189081 & -2.1891280 & 1.127959 & 1.1284628 \\
\hline & & 0.0 & -2.412287 & -2.4121919 & 1.689593 & 1.6902300 \\
\hline & & 0.6 & -3.178401 & -3.1782320 & 3.958697 & 3.9593387 \\
\hline & 5 & 0.6 & -5.768535 & -5.7687145 & 3.874307 & 3.8749347 \\
\hline \multirow[t]{3}{*}{1.9} & 0 & -0.2 & -1.073300 & -1.0734818 & 1.278189 & 1.2783778 \\
\hline & 1 & -0.2 & -2.107985 & -2.1080359 & 1.180071 & 1.1812444 \\
\hline & 5 & 0.6 & -5.977131 & -5.977046 & 3.935870 & 3.9357910 \\
\hline
\end{tabular}

\section{Conclusion}

The problem has dealt with the two dimensional heat and mass transfer free convection flow of an MHD NonNewtonian power-law fluid along stretching sheet in the presence of magnetic field with thermal radiation. From the present investigation we can make the following conclusions:

1. For the increase of the suction parameter $\left(f_{w}\right)$ the velocity, temperature and concentration significantly decrease. So, suction stabilizes the velocity, temperature and concentration field quickly to prevent the boundary layer separation.

2. Larger values of buoyancy parameter $(\lambda)$ can be used to control the temperature and concentration boundary layers.

3. The momentum boundary layer and the temperature boundary layer thickness reduce as a result of increasing radiation.

4. The presence of a heavier species (large $S c$ ) in the flow field decrease the concentration in the boundary layer.

5. Using magnetic field we can control the flow characteristics and it has significant effect on heat and mass transfer.

6. Drag of the pseudo-plastics along a stretched surface decreases as the velocity index increases.

7. The heat transfer rate of dilatant fluids are greater than pseudo-plastic fluids for a decelerated surface flow while the opposite behavior is visible for accelerated surface flow.

\section{References}

1. Sakiadis B. C., 1961. Boundary layer behavior on continuous solid surfaces: I. The Boundary Layer Equations for Two-Dimensional and Axisymmetric Flow, AICHE J. 7, 26-28.

2. Elbashbeshy E. M. A., 1998. Heat Transfer over a Stretching Surface with Variable Surface Heat Flux, J. Phys. D: Appl.Phys. 31,1951-1954.

3. Vajravelu K., A. Hadjinicolaou,1997. Convective Heat Transfer in an Electrically Conducting Fluid at a Stretching Surface with Uniform Free Stream, Int. J. Eng. Sci. 35, 12-13, 1237-1244.

4. Glauert M.B., 1961. A Study of the Magnetohydrodynamic Boundary Layer on a Flat Plate, J. Fluid Mech. 10, 276-288.

5. Rajgopal K.R., T.Y. Na, A.S. Gupta,1984. Flow of a Viscoelastic Fluid over a Stretching Sheet, Rheol, ACTA 23 , 213-215.

6. Dandapat B.S., A.S. Gupta, 2005. Flow and Heat Transfer in a Viscoelastic Fluid Over a Stretching Sheet, Int. J. Non-Linear Mech. 40. 215-219.

7. Datti P. S., K. V. Prasad, M. S. Abel, A. Joshi, 2005. MHD Viscoelastic Fluid Flow Over a Non-Isothermal Stretching Sheet, Int. J. Eng. Sci. 42, 935-946.

8. Rahaman M. M., M. A. Sattar, 2006. Magnetohydro-dynamic Convective Flow of a Micropolar Fluid Past a Continuously Moving Vertical Porous Plate in the Presence of Heat Generation/Absorption, ASME J. Heat Transfer 128,142-152.

9. Raptis A., 1998. Flow of a Micropolar FluidPast a Continuously Moving Plate by the Presence of Radiation, Int. J. Heat Mass Transfer 41, 2865-2866.

10. Anderson H.I., K. H. Bech, B.S. Dandapat, 1992. Magnetohydrodynamic Flow of a Power-Law Fluid Over a Stretching Sheet, Int. J. Non-Linear Mech. 27, 926-936. 
11. Mahmod M.A.A., M.A.E. Mahmoud, 2006. Analytical Solutions of Hydromagnetic Boundary Layer Flow of Non-Newtonian Powerlaw Fluid Past a Continuously Moving Surface, ACTA Mech. 181, 83-89.

12. Chen H.T., C.K. Chen, 1988. Free Convection of Non-Newtonian Fluids Along a Vertical Plate Embedded in a Porous Medium, Trans. ASME, J. Heat Transfer 110, 257-260.

13. Chen C.H., 2008. Effects of Magnetic Field and Suction/Injection on Convective Heat Transfer on Non-Newtonian Power-Law Fluids Past a Power-Law Stretched Sheet with Surface heat Flux, Int. J. Thermal Sci. 47, 954-961.

14. El-Hakiem M.A., 2008. Radiative Effects on Non-Darcy Natural Convection From a Heated Vertical Plate in Saturated Porous Media with Mass Transfer for Non-Newtonian Fluid, J. Porous Media 12, 89-99.

15. Chamkha A. J., H. S. Takhar and V. M. Soundalgekar, 2001. Radiation Effects on Free Convection Flow Past a Semi-Infinite Vertical Plate with Mass Transfer. Chem.Engg.J.. 84, 335-342.

16. Nachtsheim P.R.,P. Swigert,1965. Satisfaction of the Asymptotic Boundary Conditions in Numerical Solution of the System of Nonlinear Equations of Boundary Layer Type, NASA TND-3004.

17. Takhar H. S., R.S.R Gorla, V.M. Soundalgekar, 1996. Radiation Effects on MHD Free Convection Flow of a Radiating Fluid Past a Semi-Infinite Vertical Plate, Int. J. Numerical Methods for Heat and Fluid Flow 6, 77-83.

18. Ali M.M., T.S. Chen, B.F. Armaly, 1984. Natural ConvectionRadiation Interaction in Boundary-Layer Flow Over Horizontal Surfaces, AIAA Journal 22,1797-18. 
Dhaka Univ. J. Sci. 62(1): 37-44, 2014 (January) 